\title{
Incorporating Inelastic Scattering into Multislice Simulation
}

\author{
A.A. Gunawan and K.A. Mkhoyan \\ Chemical Engineering and Materials Science, University of Minnesota, Minneapolis, MN 55455
}

Existing Scanning/Transmission Electron Microscopy (S/TEM) image simulation algorithms such as Multislice and Bloch Wave are based exclusively on elastic scattering. At thicker specimens inelastic scattering may affect image intensity and contrasts. Previous semi-quantitative incorporation of inelastic plasmon scattering into the output of Multislice simulated diffraction patterns has improved the agreement between experimental and simulation results from $\sim 30 \%$ to less than $10 \%$ [1]. In this study, a full quantitative plasmon scattering algorithm was incorporated into multislice method [2] by allowing the wavefunction of the propagating beam to interact inelastically within each slice [3].

Crystalline Si samples with 4 x $4 \mathrm{~nm}$ supercells oriented along $<100>$ crystallographic direction with various thicknesses were used in this simulation. STEM operated at $100 \mathrm{kV}$ accelerating voltage and $11.4 \mathrm{mrad}$ convergence angle giving about $2 \AA$ diameter probe was used here. High-angle annular darkfield (HAADF) STEM images and Electron Energy Loss Spectroscopy (EELS) signals were calculated. For HAADF-STEM images 54 - $150 \mathrm{mrad}$ detector was used and $20 \mathrm{mrad}$ collection angles was used for EELS.

The modified "inelastic-elastic" multislice method first takes into account the plasmon scattering by calculating the energy loss for each scattering angles before undergoing the elastic scattering through transmission operation. Then beam propagates to the next slice using propagation operator. Simulated EELS spectra in Si using new "inelastic-elastic" multislice method for thicknesses of 42, 74, 83, and $130 \mathrm{~nm}$ are shown in Figure 1 comparable with early experiments performed under similar conditions [4]. An energy dispersion of $1 \mathrm{eV}$ (for energy loss up to $60 \mathrm{eV}$ ) was used in the simulation to monitor single, double, and triple plasmon scattering in these Si samples. A good agreement was achieved for the range of specimen thicknesses.

Figure 2 shows simulated HAADF-STEM images under different multislice modes. The simulated image of the $\mathrm{Si}(100)$ unit cell for $42 \mathrm{~nm}$ thick sample without inelastic scattering is shown in Figure 2(a). For efficient computational time, only a quarter of the unit cell was studied (Figure 2(b)). The "inelastic-elastic" simulated image of the same area is shown in Figure 2(c). The contribution from inelastic scattering is still small at this thickness resulting in only small changes in the image intensity. Hence, Figure 2(b) and (c) look somewhat similar. Additionally, the inelastic scattering intensity collected by the HAADF detector can be analyzed separately using this "inelastic-elastic" multislice algorithm (Figure 2(d)). Thicker specimens are expected to display more significant contributions to the HAADF-STEM signals. The calculated image intensity and contrast could be improved considerably by incorporating these inelastic scatterings into multislice algorithm [5].

\section{References:}

[1] K.A. Mkhoyan et al., Phys. Rev. Lett, 100 (2008) 025503.

[2] E.J. Kirkland, Advanced Computing in Electron Microscopy, 2nd edition, Springer (2010).

[3] A.A. Gunawan and K. A. Mkhoyan, in preparation.

[4] K.A. Mkhoyan et al., Ultramicroscopy, 107 (2006) 345. 
[5] This work was partially supported by MRSEC Program of the National Science Foundation under Award Number DMR-0819885.

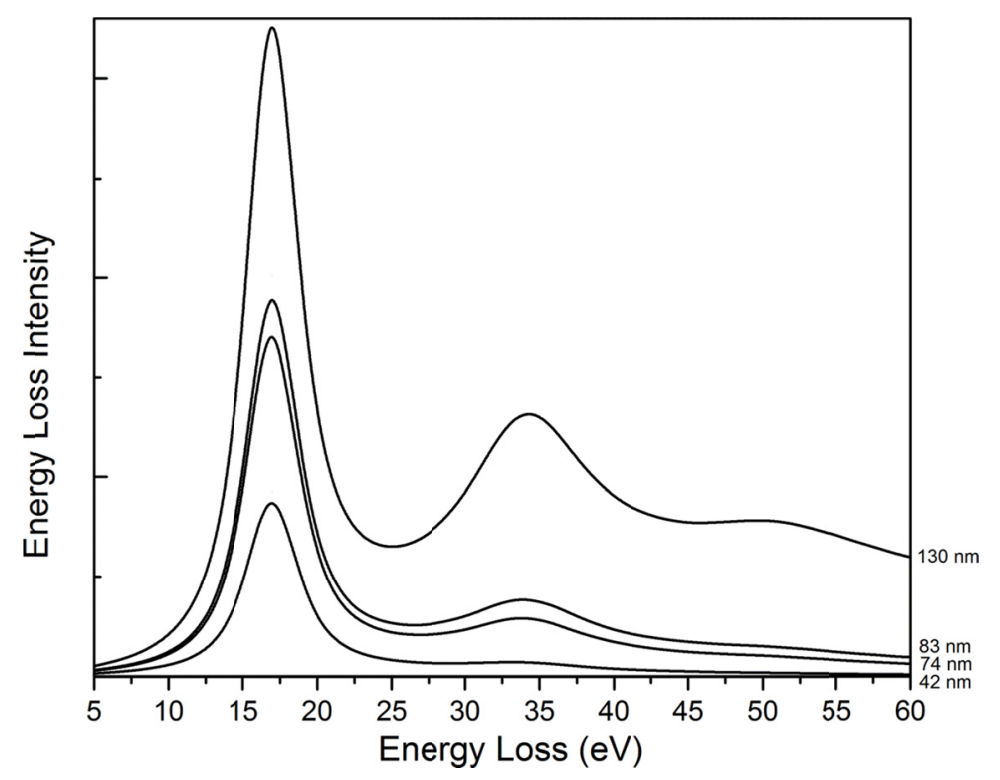

Figure 1. Simulated EELS spectra of $\mathrm{Si}(100)$ using the modified "inelastic-elastic" multislice method. The collection angle is 20 mrad with samples thicknesses of 42, 74, 83, and $130 \mathrm{~nm}$.
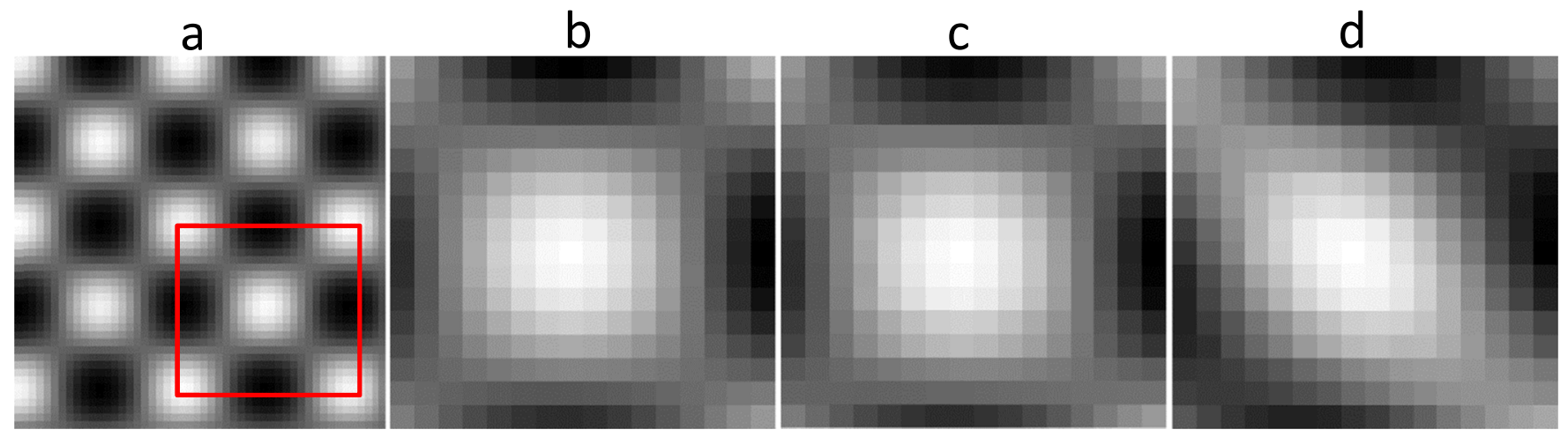

Figure 2. Simulated HAADF-STEM images of $42 \mathrm{~nm}$ thick Si(100) crystal. (a) HAADF-STEM image of a unit cell calculated using only elastic scatterings, (b) close-up of the image in (a) (red-boxed region), (c) HAADF-STEM image of the same region as in (b) calculated using "inelastic-elastic" method, and (d) HAADF-STEM image of the same region as in (b) calculated using only inelastic scattered electrons. The images are individually normalized to gray-scale. 